\title{
Specimen Ultra-High-Field Magnetic Resonance Imaging
}

National Cancer Institute

\section{Source}

National Cancer Institute. Specimen Ultra-High-Field Magnetic Resonance Imaging. NCI Thesaurus. Code C116460.

A type of magnetic resonance imaging that uses very high field stength to provide a very detailed image of the targeted tissue. 